\title{
Editorial
}

\section{Sign-Changing Solutions to Equations of Elliptic Type}

\section{Wenming Zou, ${ }^{1}$ Thomas Bartsch, ${ }^{2}$ Norimichi Hirano, ${ }^{3}$ Martin Schechter, ${ }^{4}$ and Zhi-Qiang Wang ${ }^{5}$}

\footnotetext{
${ }^{1}$ Department of Mathematical Sciences, Tsinghua University, Beijing 100084, China

${ }^{2}$ Mathematisches Institut, University Giessen, Arndtstr. 2, 35392 Giessen, Germany

${ }^{3}$ Graduate School of Environment and Information Science, Yokohama National University, Tokiwadai, Yokohama 240-8501, Japan

${ }^{4}$ Mathematics Department, University of California, Irvine, CA 92697-3875, USA

${ }^{5}$ Department of Mathematics and Statistics, Utah State University, Logan, UT 84322-4205, USA
}

Correspondence should be addressed to Wenming Zou, wzou@math.tsinghua.edu.cn

Received 21 March 2010; Accepted 21 March 2010

Copyright (C) 2010 Wenming Zou et al. This is an open access article distributed under the Creative Commons Attribution License, which permits unrestricted use, distribution, and reproduction in any medium, provided the original work is properly cited.

The equation of elliptic type is one of the most important equations which is closely related to the real world. The studies of the existence of solutions for elliptic problems under various boundary conditions have received much attention over the last decades. Among them, the number of solutions, the geometrical and topological properties for those solutions are essentially the subject matter on this line. In the recent years, there has been an increasing interest to develop a theory by which one can obtain much more information on the solutions. In particular, the nodal properties of solutions to elliptic equations are becoming increasing popular. The central theme of the current special issue is about the sign-changing solutions to equations of elliptic type.

The first paper of this special issue addresses the multiplicity result for antisymmetric sign changing solutions of an elliptic equations defined on a smooth compact-connected Riemannian manifold without boundary and with dimension greater than one. They obtain the multiplicity of the sign-changing solutions which change sign exactly once. In particular, the number of solutions depends on the Poincare polynomial.

The second paper presents the study of the asymptotic behaviour for the second eigenvalue of the $p$-Laplacian operator as $p$ goes to 1 . In the particular case of a planar disc, it is possible to show that the second eigenfunctions are nonradial if $p$ is close enough to 1 . The main goal of the third paper is to present multiple solutions results for elliptic inclusions of Clarke's gradient type under Dirichlet boundary condition involving the $p$-Laplacian which, in general, depend on two parameters. The authors prove the existence of multiple constant-sign 
and sign-changing (nodal) solutions for parameters specified in terms of the Fucik spectrum of the $p$-Laplacian.

The fourth paper is devoted to the quasilinear elliptic equation defined on the entire space. The author proves that the equation admits a positive ground-state solution in the entire space. Combining with some ideas due to Cerami-Solomini-Struwe, one nodal solution is obtained. In the fifth paper, by using the Fountain Theorem, the authors obtain infinitely many solutions for a class of superlinear elliptic problems with the Robin boundary value under weaker conditions than in the earlier papers. The authors of the sixth paper study the large solutions of the quasilinear elliptic system of competitive type with boundary blow-up conditions, including the existence, uniqueness, and asymptotic behavior of positive solutions via the methods of upper and lower solutions.

Wenming Zou

Thomas Bartsch

Norimichi Hirano

Martin Schechter

Zhi-Qiang Wang 


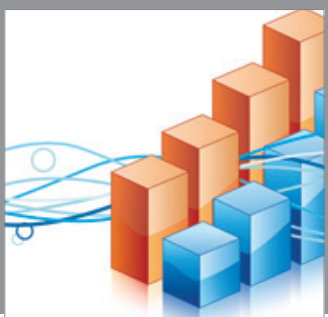

Advances in

Operations Research

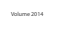

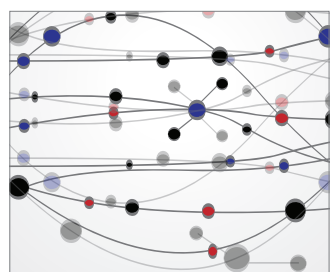

\section{The Scientific} World Journal
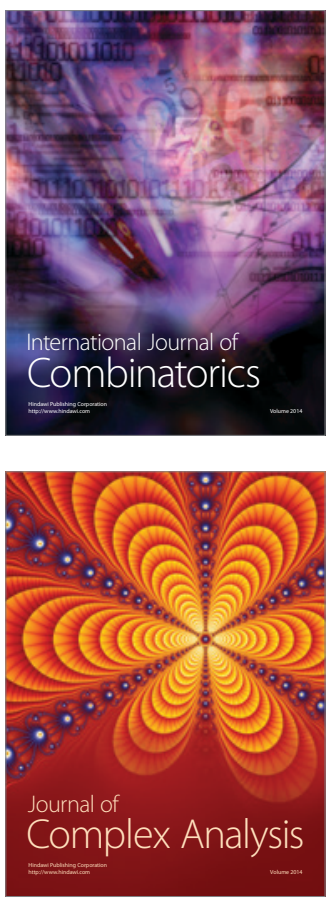

International Journal of

Mathematics and

Mathematical

Sciences
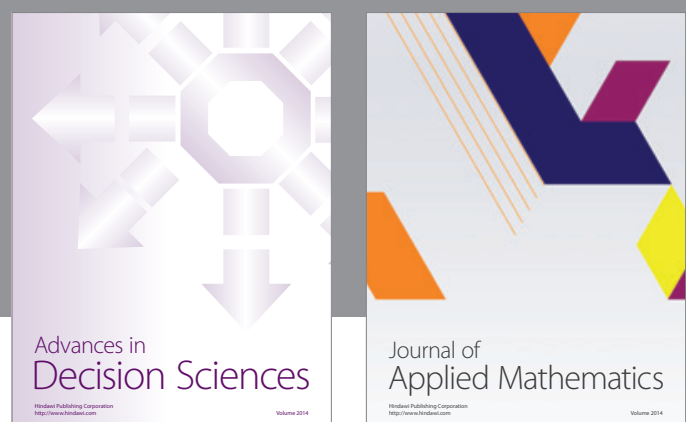

Journal of

Applied Mathematics
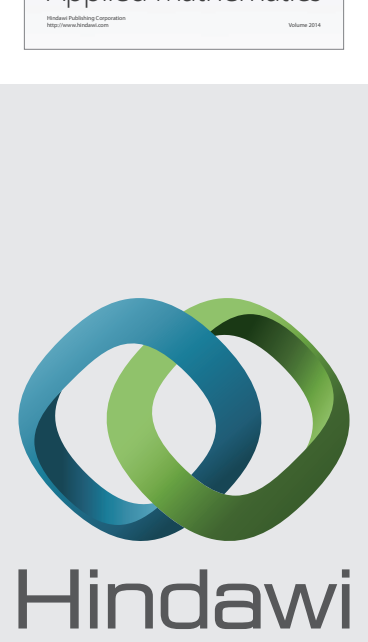

Submit your manuscripts at http://www.hindawi.com
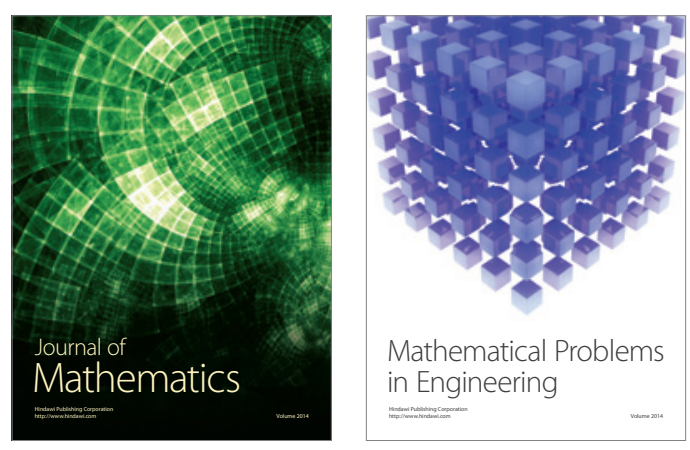

Mathematical Problems in Engineering
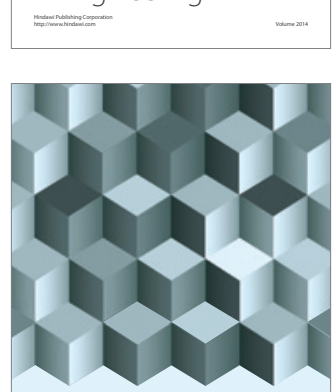

Journal of

Function Spaces
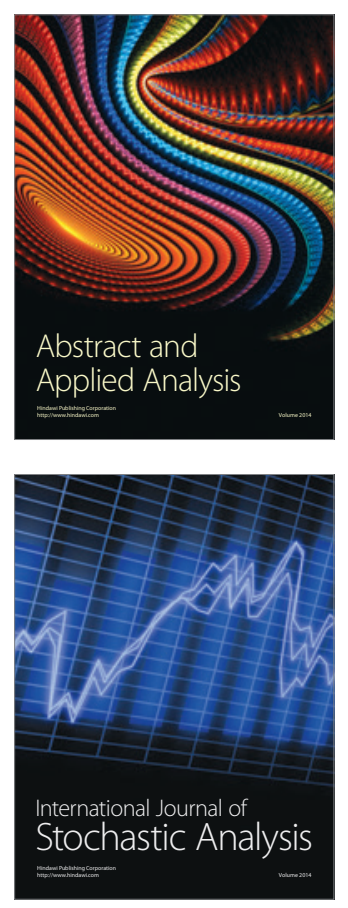

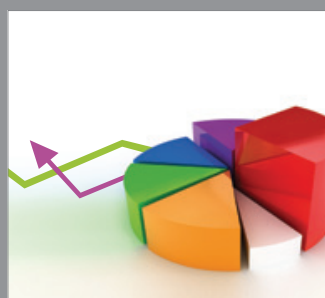

ournal of

Probability and Statistics

Promensencen
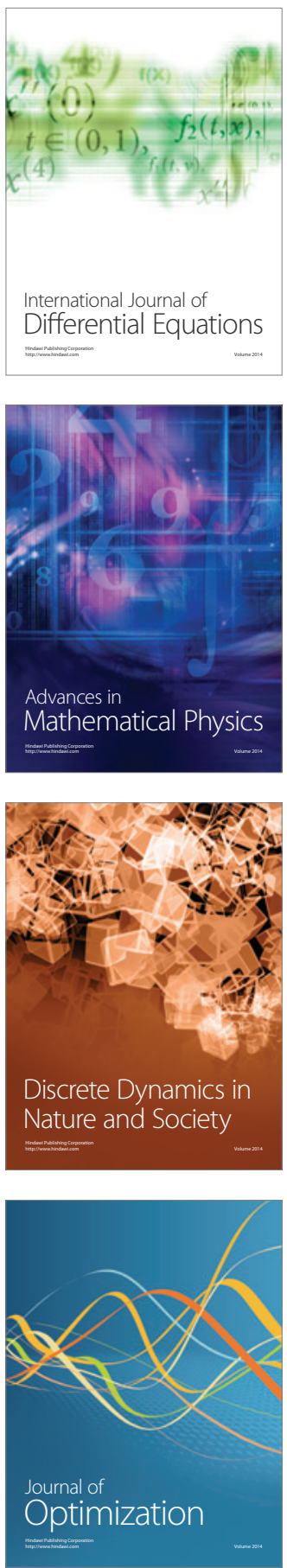